\title{
Dimensions of non-differentiability points of Cantor functions
}

\author{
by \\ YuAnyuan YaO, Yunxiu Zhang and Wenxia Li (Shanghai)
}

\begin{abstract}
For a probability vector $\left(p_{0}, p_{1}\right)$ there exists a corresponding self-similar Borel probability measure $\mu$ supported on the Cantor set $C$ (with the strong separation property) in $\mathbb{R}$ generated by a contractive similitude $h_{i}(x)=a_{i} x+b_{i}, i=0,1$. Let $S$ denote the set of points of $C$ at which the probability distribution function $F(x)$ of $\mu$ has no derivative, finite or infinite. The Hausdorff and packing dimensions of $S$ have been found by several authors for the case that $p_{i}>a_{i}, i=0,1$. However, when $p_{0}<a_{0}$ (or equivalently $p_{1}<a_{1}$ ) the structure of $S$ changes significantly and the previous approaches fail to be effective any more. The present paper is devoted to determining the Hausdorff and packing dimensions of $S$ for the case $p_{0}<a_{0}$.
\end{abstract}

1. Introduction. Let $C \subset \mathbb{R}$ be the unique nonempty compact set invariant under $h_{0}, h_{1}$ :

$$
C=h_{0}(C) \cup h_{1}(C),
$$

where $h_{j}(x)=a_{j} x+b_{j}, j=0,1$, with $0<a_{j}<1$. The set $C$ is also termed the Cantor set, or the self-similar set determined by $h_{0}$ and $h_{1}$. Without loss of generality we shall assume that $b_{0}=0, a_{1}+b_{1}=1$ (so $h_{0}(0)=0$ and $\left.h_{1}(1)=1\right)$. We furthermore assume that the images $h_{j}([0,1]), j=0,1$, are pairwise disjoint (i.e. the $h_{j}$ 's satisfy the strong separation condition). Let

$$
\beta=1-a_{0}-a_{1}>0
$$

be the length of the gap between $h_{0}([0,1])$ and $h_{1}([0,1])$. It is well known (cf. $[4,6]$ ) that $\operatorname{dim}_{\mathrm{H}} C=\operatorname{dim}_{\mathrm{P}} C=\operatorname{dim}_{\mathrm{B}} C=\xi$ and $0<\mathcal{H}^{\xi}(C)<\infty$ where $\xi$ is given by

$$
a_{0}^{\xi}+a_{1}^{\xi}=1 .
$$

The set $C$ has a natural symbolic representation defined as follows. Let

2010 Mathematics Subject Classification: 28A80, 28A78.

Key words and phrases: Hausdorff dimension, Cantor function, non-differentiability point, self-similar measure, distribution function.

W. X. Li is the corresponding author. 


$$
\Sigma^{*}=\bigcup_{n=1}^{\infty}\{0,1\}^{n} \text { and } \quad \Sigma^{\mathbb{N}}=\{0,1\}^{\mathbb{N}},
$$

i.e. $\Sigma^{*}$ is the family of all finite strings $j_{1} \ldots j_{n}$ with entries $j_{i}$ from $\{0,1\}$ and $\Sigma^{\mathbb{N}}$ denotes the family of all infinite strings $j_{1} j_{2} \ldots$ with entries $j_{i}$ from $\{0,1\}$. For $\omega=j_{1} j_{2} \ldots \in \Sigma^{\mathbb{N}}$ and a positive integer $n$, let $\omega \mid n=j_{1} \ldots j_{n}$ denote the truncation of $\omega$ to the $n$th place. Finally, we define $\pi: \Sigma^{\mathbb{N}} \rightarrow C$ by

$$
\{\pi(\omega)\}=\bigcap_{n=1}^{\infty} h_{j_{1}} \circ \cdots \circ h_{j_{n}}([0,1])
$$

for $\omega=j_{1} j_{2} \ldots \in \Sigma^{\mathbb{N}}$. It is easy to check that for $\omega=j_{1} j_{2} \ldots \in \Sigma^{\mathbb{N}}$,

$$
\pi(\omega)=\lim _{n \rightarrow \infty} h_{\omega \mid n}(0)=b_{j_{1}}+\sum_{n=2}^{\infty} a_{j_{1}} \ldots a_{j_{n-1}} b_{j_{n}}
$$

where $b_{0}=0$ and $b_{1}=1-a_{1}$. It is well known that $C$ can be symbolically represented as $C=\pi\left(\Sigma^{\mathbb{N}}\right)$. Thus, each point of $C$ can be encoded by a unique element from $\Sigma^{\mathbb{N}}$. Throughout this paper, by $\tilde{t}=t_{1} t_{2} \ldots$ we denote the code of $t \in C$, i.e., $\tilde{t}=t_{1} t_{2} \ldots \in \Sigma^{\mathbb{N}}$ with $\pi(\tilde{t})=t$. Alternatively, we have

$$
C=\bigcap_{n=1}^{\infty} \bigcup_{\omega \in\{0,1\}^{n}} h_{\omega}([0,1])
$$

where $h_{\omega}:=h_{j_{1}} \circ \cdots \circ h_{j_{n}}$ for $\omega=j_{1} \ldots j_{n} \in\{0,1\}^{n}$. The endpoints of $h_{\omega}([0,1])$ for $\omega \in \Sigma^{*}$ will be called the endpoints of $C$. Obviously any endpoint $e$ of $C$ lies in $C$ and except for a finite number of terms, its coding $\tilde{e}$ consists of either only the digit 0 if $e$ is the left endpoint of some $h_{\omega}([0,1])$, or only the digit 1 if $e$ is the right endpoint of some $h_{\sigma}([0,1])$. Moreover, for each non-endpoint $t$ of $C$ its code $\tilde{t}$ consists of infinitely many 0 s and infinitely many $1 \mathrm{~s}$.

Let $\mu$ be the self-similar Borel measure on $C$ corresponding to the probability vector $\left(p_{0}, p_{1}\right)$ where each $p_{i}>0$ and $p_{0}+p_{1}=1$, i.e., the measure satisfying

$$
\mu(A)=p_{0} \mu\left(h_{0}^{-1}(A)\right)+p_{1} \mu\left(h_{1}^{-1}(A)\right) \text { for any Borel set } A,
$$

and so

$$
\mu\left(h_{\omega}[0,1]\right)=\prod_{i=1}^{k} p_{j_{i}}=: p_{\omega} \quad \text { for any } \omega=j_{1} \ldots j_{k} \in\{0,1\}^{k}, k \in \mathbb{N} .
$$

Obviously, $\mu$ is atomless. Consider the distribution function of the probability measure $\mu$, also called the Cantor function or a self-affine "devil's staircase" function:

$$
F(x)=\mu([0, x]), \quad x \in[0,1]
$$


Then $F(x)$ is a non-decreasing continuous function with $F(0)<F(1)$ that is constant off the support of $\mu$. It is easy to check that the derivative of $F(x)$ is zero for all $x \in[0,1] \backslash C$. In particular, the set $S$ of points of non-differentiability of $F(x)$, that is, those $x$ where

$$
\lim _{\delta \rightarrow 0} \frac{F(x+\delta)-F(x)}{\delta}
$$

does not exist either as a finite number or $\infty$, has Lebesgue measure 0 . It has been shown that $F^{\prime}(x)=\infty$ for $\mu$-a.e. $x \in C$ and so $\mu(S)=0$ (cf. [8]). Some further results on the size of $S$ have also been obtained.

(I) When $\left(p_{0}, p_{1}\right)=\left(a_{0}^{\xi}, a_{1}^{\xi}\right)$, we have $\operatorname{dim}_{\mathrm{H}} S=\xi^{2}=\left(\operatorname{dim}_{\mathrm{H}} C\right)^{2}$ and $\operatorname{dim}_{\mathrm{P}} S=\operatorname{dim}_{\mathrm{B}} S=\xi=\operatorname{dim}_{\mathrm{H}} C$ (cf. [1-3]). In this case, the corresponding $\mu$ is $\xi$-Ahlfors regular, which means that there exist $c_{1}, c_{2}>0$ such that $c_{1} \delta^{\xi} \leq$ $\mu([x-\delta, x+\delta]) \leq c_{2} \delta^{\xi}$ for all $x \in C$ and $0<\delta<1$. This curious property is extended to a more general setting. K. J. Falconer [5] proved that for $0<d<1$ if $\mu$ is a positive finite Borel $d$-Ahlfors regular measure supported by a non-empty compact subset $E$ of $\mathbb{R}$ (not necessarily the Cantor set), then $\operatorname{dim}_{\mathrm{H}} S=\left(\operatorname{dim}_{\mathrm{H}} E\right)^{2}$ and $\operatorname{dim}_{\mathrm{P}} S=\operatorname{dim}_{\mathrm{B}} S=\operatorname{dim}_{\mathrm{H}} E$.

(II) When $\left(p_{0}, p_{1}\right)$ satisfies $p_{0}>a_{0}$ and $p_{1}>a_{1}$ (so $\mu$ is not Ahlfors regular except in a special case), $\operatorname{dim}_{\mathrm{P}} S=\operatorname{dim}_{\mathrm{B}} S=\xi=\operatorname{dim}_{\mathrm{H}} C$ still holds and another approach has to be taken to determine $\operatorname{dim}_{\mathrm{H}} S$ (cf. [7, 9]). In particular, when $p_{0}=a_{0} /\left(a_{0}+a_{1}\right)$ and $p_{1}=a_{1} /\left(a_{0}+a_{1}\right), \operatorname{dim}_{\mathrm{H}} S$ was found by J. Morris [10].

Clearly, case (I) is included in case (II) since $p_{i}=a_{i}^{\xi}>a_{i}, i=0,1$. The condition $p_{i}>a_{i}, i=0,1$, implies that (cf. [9])

$$
\limsup _{\delta \rightarrow 0} \frac{F(x+\delta)-F(x)}{\delta}=\infty \quad \text { for all } x \in C .
$$

Thus, we have

$$
S=\left\{x \in C: \liminf _{\delta \rightarrow 0} \frac{F(x+\delta)-F(x)}{\delta}<\infty\right\} \quad \text { if } p_{0}>a_{0}, p_{1}>a_{1} .
$$

In particular,

(4) $\left\{x \in C: F^{\prime}(x)=\lim _{\delta \rightarrow 0} \frac{F(x+\delta)-F(x)}{\delta}=0\right\}=\emptyset \quad$ if $p_{0}>a_{0}, p_{1}>a_{1}$.

However, when $p_{0}<a_{0}$ and $p_{1}>a_{1}$ (or $p_{0}>a_{0}$ and $p_{1}<a_{1}$ ), the property (3) does not hold any more. This makes the structure of $S$ more complicated, e.g., compared to (4); we will see in Theorem 1.1 that there are massive sets of points of $C$ at which the derivative is zero in this case, and so the approaches used in the papers mentioned above are not valid any more. 
The present paper is devoted to determining the fractal dimensions of $S$ for the case $p_{0}<a_{0}$ and $p_{1}>a_{1}$. Let $s$ be such that

$$
\left(\frac{p_{0}}{a_{0}}\right)\left(\frac{p_{1}}{a_{1}}\right)^{s}=1, \quad \text { i.e., } \quad s=\frac{\log \left(a_{0} / p_{0}\right)}{\log \left(p_{1} / a_{1}\right)}>0 .
$$

We remark that $s$ can take any positive value when $p_{0}$ varies in $\left(0, a_{0}\right)$. Let $\widetilde{C}=C \backslash\{$ the endpoints of $C\}$. Since the Cantor set $C$ has only countably many endpoints, removing the endpoints of $C$ from $S$ does not affect the size of $S$. In the following, we still use the notation $S$ to denote the non-endpoints of $C$ for which (2) does not exist either as a finite number or $\infty$. For $t \in \widetilde{C}$, let

$$
N_{n}(t, 0)=\#\left\{1 \leq l \leq n: t_{l}=0\right\} \quad \text { and } \quad N_{n}(t, 1)=\#\left\{1 \leq l \leq n: t_{l}=1\right\}
$$

where, as mentioned above, $\tilde{t}=t_{1} t_{2} \ldots \in \Sigma^{\mathbb{N}}$ is the code of $t$. We will first show that

$$
\begin{aligned}
&\left\{t \in \widetilde{C}: \liminf _{n \rightarrow \infty} \frac{N_{n}(t, 0)}{n}<\frac{1}{s+1}\right.\left.<\limsup _{n \rightarrow \infty} \frac{N_{n}(t, 0)}{n}\right\} \subseteq S \\
& \subseteq\left\{t \in \widetilde{C}: \liminf _{n \rightarrow \infty} \frac{N_{n}(t, 0)}{n} \leq \frac{1}{s+1}\right\} .
\end{aligned}
$$

By applying the results on divergence points in $[11,12]$ we obtain the following theorems.

TheOREM 1.1. Let $p_{0}<a_{0}$ and $p_{1}>a_{1}$. Let $s$ and $\xi$ be defined as in (5) and (1). Let

$$
S_{0}=\left\{t \in C: F^{\prime}(t)=0\right\} \quad \text { and } \quad S_{\infty}=\left\{t \in C: F^{\prime}(t)=\infty\right\} .
$$

Then $C=S \cup S_{0} \cup S_{\infty}$, and

$$
\begin{aligned}
\operatorname{dim}_{\mathrm{H}} S_{0} & \geq \max _{p \in\left[(s+1)^{-1}, 1\right]} \frac{p \log p+(1-p) \log (1-p)}{p \log a_{0}+(1-p) \log a_{1}}, \\
\operatorname{dim}_{\mathrm{H}} S_{\infty} & \geq \max _{p \in\left[0,(s+1)^{-1}\right]} \frac{p \log p+(1-p) \log (1-p)}{p \log a_{0}+(1-p) \log a_{1}} .
\end{aligned}
$$

In particular, $\operatorname{dim}_{\mathrm{H}} S_{0}=\xi$ if $(s+1)^{-1} \leq a_{0}^{\xi}$, and $\operatorname{dim}_{\mathrm{H}} S_{\infty}=\xi$ if $(s+1)^{-1} \geq a_{0}^{\xi}$.

TheOrem 1.2. Let $p_{0}<a_{0}$ and $p_{1}>a_{1}$. Let $s$ and $\xi$ be defined as in (5) and (1). If $1 /(s+1) \leq a_{0}^{\xi}$, then

$$
\operatorname{dim}_{\mathrm{H}} S=\frac{s \log s-(s+1) \log (s+1)}{\log a_{0}+s \log a_{1}}, \quad \operatorname{dim}_{\mathrm{P}} S=\xi .
$$

2. Proofs. In this section, we prove Theorems 1.1 and 1.2 . We first characterize $S$ by analyzing for each $t \in S$ the behavior of $N_{n}(t, 0) / n$, the frequency of the digit 0 occurring in the first $n$ terms of the code of $t$. The desired results are then obtained by applying the results from [11, 12]. For 
$t \in \widetilde{C}$, we denote by $z(t, n)$ the position of the $n$th occurrence of 0 in $\tilde{t}$ (recall we use $\tilde{t}$ to denote the code of $t$ ) and by $y(t, n)$ the position of the $n$th occurrence of 1 in $\tilde{t}$. Since $t \in \widetilde{C}$ (the non-endpoint of $C$ ), we have $n \leq z(t, n), y(t, n)<\infty$ and $\lim _{n \rightarrow \infty} y(t, n)=\lim _{n \rightarrow \infty} z(t, n)=\infty$.

Lemma 2.1. For each $t \in \widetilde{C}$ we have

$$
\begin{array}{ll}
\limsup _{n \rightarrow \infty} \frac{N_{n}(t, 0)}{n}=\limsup _{n \rightarrow \infty} \frac{n}{z(t, n)}, & \liminf _{n \rightarrow \infty} \frac{N_{n}(t, 0)}{n}=\liminf _{n \rightarrow \infty} \frac{n}{z(t, n)}, \\
\limsup _{n \rightarrow \infty} \frac{N_{n}(t, 1)}{n}=\limsup _{n \rightarrow \infty} \frac{n}{y(t, n)}, & \liminf _{n \rightarrow \infty} \frac{N_{n}(t, 1)}{n}=\liminf _{n \rightarrow \infty} \frac{n}{y(t, n)} .
\end{array}
$$

Proof. It is obvious that

$$
\begin{aligned}
& \limsup _{n \rightarrow \infty} \frac{N_{n}(t, 0)}{n} \geq \limsup _{n \rightarrow \infty} \frac{N_{z(t, n)}(t, 0)}{z(t, n)}=\limsup _{n \rightarrow \infty} \frac{n}{z(t, n)}, \\
& \liminf _{n \rightarrow \infty} \frac{N_{n}(t, 0)}{n} \leq \liminf _{n \rightarrow \infty} \frac{N_{z(t, n)}(t, 0)}{z(t, n)}=\liminf _{n \rightarrow \infty} \frac{n}{z(t, n)},
\end{aligned}
$$

On the other hand, note that $z\left(t, N_{n}(t, 0)\right) \leq n<z\left(t, N_{n}(t, 0)+1\right)$ for all $n \in \mathbb{N}$. Thus, for all $n \in \mathbb{N}$,

$$
\frac{N_{n}(t, 0)}{z\left(t, N_{n}(t, 0)+1\right)}<\frac{N_{n}(t, 0)}{n} \leq \frac{N_{n}(t, 0)}{z\left(t, N_{n}(t, 0)\right)} .
$$

Noting that $\lim _{n \rightarrow \infty} N_{n}(t, 0)=\infty$, this gives the opposite inequalities in (6) and (7). The other two equalities can be deduced in the same way.

From Lemma 2.1 it follows that for each $t \in \widetilde{C}$,

(8) $\quad \limsup _{n \rightarrow \infty} \frac{z(t, n)}{n}>s+1$ if and only if $\liminf _{n \rightarrow \infty} \frac{y(t, n)}{n}<\frac{s+1}{s}$,

(9) $\quad \limsup _{n \rightarrow \infty} \frac{z(t, n)}{n}<s+1$ if and only if $\liminf _{n \rightarrow \infty} \frac{y(t, n)}{n}>\frac{s+1}{s}$.

In fact, with the convention $0^{-1}=\infty, \infty^{-1}=0$ and by Lemma 2.1 we have

$$
\begin{aligned}
\limsup _{n \rightarrow \infty} \frac{z(t, n)}{n} & =\left(\liminf _{n \rightarrow \infty} \frac{n}{z(t, n)}\right)^{-1}=\left(\liminf _{n \rightarrow \infty} \frac{N_{n}(t, 0)}{n}\right)^{-1} \\
& =\left(1-\limsup _{n \rightarrow \infty} \frac{N_{n}(t, 1)}{n}\right)^{-1}=\left(1-\limsup _{n \rightarrow \infty} \frac{n}{y(t, n)}\right)^{-1} \\
& =\left(1-\frac{1}{\liminf _{n \rightarrow \infty} y(t, n) / n}\right)^{-1}
\end{aligned}
$$

which leads to (8) and (9). 
LEMma 2.2. For each $t \in \widetilde{C}$ we have

$$
\begin{aligned}
& \liminf _{n \rightarrow \infty} \frac{y(t, n)}{n}>\frac{s+1}{s} \Rightarrow \lim _{x \rightarrow t} \frac{F(x)-F(t)}{x-t}=0, \\
& \liminf _{n \rightarrow \infty} \frac{y(t, n)}{n}<\frac{s+1}{s} \Rightarrow \limsup _{x \rightarrow t} \frac{F(x)-F(t)}{x-t}=\infty, \\
& \limsup _{n \rightarrow \infty} \frac{y(t, n)}{n}>\frac{s+1}{s} \Rightarrow \liminf _{x \rightarrow t} \frac{F(x)-F(t)}{x-t}=0 .
\end{aligned}
$$

Proof. (I) If $\liminf \inf _{n \rightarrow \infty} y(t, n) / n>(s+1) / s$, then there exist $\epsilon>0$ and $k \in \mathbb{N}$ such that

$$
y(t, n)>n\left(1+\frac{1}{s}\right)+n \epsilon \quad \text { for } n \geq k .
$$

Let $u$ be the distance between $t$ and $[0,1] \backslash \bigcup_{\omega \in\{0,1\}^{l}} h_{\omega}([0,1])$ with $l=y(t, k)$. Then $u>0$. Let $x$ be a point in $(t-u, t)$. Then $t, x \in h_{\tilde{t} \mid(y(t, n)-1)}([0,1])$, but $x \notin h_{\tilde{t} \mid y(t, n)}([0,1])$ with some $n>k$. Therefore (recall that $\beta=1-a_{0}-a_{1}$ ),

$$
\frac{F(x)-F(t)}{x-t} \leq \frac{\prod_{i=1}^{y(t, n)-1} p_{t_{i}}}{\beta \prod_{i=1}^{y(t, n)-1} a_{t_{i}}}=\frac{a_{1}}{\beta p_{1}}\left(\frac{p_{1}}{a_{1}}\right)^{n}\left(\frac{p_{0}}{a_{0}}\right)^{y(t, n)-n} \leq \frac{a_{1}}{\beta p_{1}}\left(\frac{p_{0}}{a_{0}}\right)^{n \epsilon}
$$

by $(10)$. Hence $\lim _{x \uparrow t}(F(x)-F(t)) /(x-t)=0$.

On the other hand, by (9) there exist $\epsilon>0$ and $k \in \mathbb{N}$ such that

$$
z(t, n)<n(s+1)-n \epsilon \quad \text { for } n \geq k .
$$

Let $u$ be the distance between $t$ and $[0,1] \backslash \bigcup_{\omega \in\{0,1\}^{l}} h_{\omega}([0,1])$ with $l=z(t, k)$. Then $u>0$. Let $x$ be a point in $(t, t+u)$. Then $t, x \in h_{\tilde{t} \mid(z(t, n)-1)}([0,1])$, but $x \notin h_{\tilde{t} \mid z(t, n)}([0,1])$ for some $n>k$. Therefore,

$$
\frac{F(x)-F(t)}{x-t} \leq \frac{\prod_{i=1}^{z(t, n)-1} p_{t_{i}}}{\beta \prod_{i=1}^{z(t, n)-1} a_{t_{i}}}=\frac{a_{0}}{\beta p_{0}}\left(\frac{p_{0}}{a_{0}}\right)^{n}\left(\frac{p_{1}}{a_{1}}\right)^{z(t, n)-n} \leq \frac{a_{0}}{\beta p_{0}}\left(\frac{p_{1}}{a_{1}}\right)^{-n \epsilon},
$$

by (11). Hence $\lim _{x \downarrow t}(F(x)-F(t)) /(x-t)=0$.

(II) If $\liminf _{n \rightarrow \infty} y(t, n) / n<(s+1) / s$, then there exist $\epsilon>0$ and a sequence $\left\{n_{k}\right\}_{k \geq 1}$ of integers with $n_{k} \uparrow \infty$ such that

$$
y\left(t, n_{k}\right)<n_{k}\left(1+\frac{1}{s}\right)-n_{k} \epsilon
$$

Now take $x_{k}$ as the left endpoint of the interval $h_{\tilde{t} \mid\left(y\left(t, n_{k}\right)-1\right)}([0,1])$. Then, by (12),

$$
\begin{aligned}
\frac{F\left(x_{k}\right)-F(t)}{x_{k}-t} & \geq \frac{p_{0} \prod_{i=1}^{y\left(t, n_{k}\right)-1} p_{t_{i}}}{\prod_{i=1}^{y\left(t, n_{k}\right)-1} a_{t_{i}}}=\frac{p_{1}^{n_{k}-1} p_{0}^{y\left(t, n_{k}\right)-n_{k}+1}}{a_{1}^{n_{k}-1} a_{0}^{y\left(t, n_{k}\right)-n_{k}}} \\
& \geq a_{1} p_{0} p_{1}^{-1}\left(\frac{p_{0}}{a_{0}}\right)^{-n_{k} \epsilon}
\end{aligned}
$$


which gives $\lim \sup _{\delta \uparrow 0}(F(t+\delta)-F(t)) / \delta=\infty$. On the other hand, by (8) there exist $\epsilon>0$ and a sequence $\left\{n_{k}\right\}_{k \geq 1}$ of integers with $n_{k} \uparrow \infty$ such that

$$
z\left(t, n_{k}\right)>n_{k}(s+1)+n_{k} \epsilon .
$$

By letting $x_{k}$ be the right endpoint of the interval $h_{\tilde{t} \mid\left(z\left(t, n_{k}\right)-1\right)}([0,1])$ we have

$$
\begin{aligned}
\frac{F\left(x_{k}\right)-F(t)}{x_{k}-t} & \geq \frac{p_{1} \prod_{i=1}^{z\left(t, n_{k}\right)-1} p_{t_{i}}}{\prod_{i=1}^{z\left(t, n_{k}\right)-1} a_{t_{i}}}=\frac{p_{0}^{n_{k}-1} p_{1}^{z\left(t, n_{k}\right)-n_{k}+1}}{a_{0}^{n_{k}-1} a_{1}^{z\left(t, n_{k}\right)-n_{k}}} \\
& \geq a_{0} p_{1} p_{0}^{-1}\left(\frac{p_{1}}{a_{1}}\right)^{n_{k} \epsilon},
\end{aligned}
$$

yielding $\lim \sup _{\delta \downarrow 0}(F(t+\delta)-F(t)) / \delta=\infty$.

(III) This can be proved in the same way as in (II). The details are left to the reader.

From Lemma 2.2 it follows that

$$
\begin{aligned}
\left\{t \in \widetilde{C}: \liminf _{n \rightarrow \infty} \frac{y(t, n)}{n}<\frac{s+1}{s}\right. & \left.<\limsup _{n \rightarrow \infty} \frac{y(t, n)}{n}\right\} \subseteq S \\
& \subseteq\left\{t \in \widetilde{C}: \liminf _{n \rightarrow \infty} \frac{y(t, n)}{n} \leq \frac{s+1}{s}\right\},
\end{aligned}
$$

and so

$$
\begin{aligned}
\left\{t \in \widetilde{C}: \liminf _{n \rightarrow \infty} \frac{N_{n}(t, 0)}{n}\right. & \left.<\frac{1}{s+1}<\limsup _{n \rightarrow \infty} \frac{N_{n}(t, 0)}{n}\right\} \subseteq S \\
& \subseteq\left\{t \in \widetilde{C}: \liminf _{n \rightarrow \infty} \frac{N_{n}(t, 0)}{n} \leq \frac{1}{s+1}\right\},
\end{aligned}
$$

by Lemma 2.1. For a sequence $\left\{x_{n}\right\}$ in a metric space $X$, we let $A\left(\left\{x_{n}\right\}\right)$ denote the set of accumulation points of the sequence $\left\{x_{n}\right\}$, i.e., $A\left(\left\{x_{n}\right\}\right)=\left\{x \in X\right.$ : there exists a subsequence $\left\{x_{n_{k}}\right\}$ such that $\left.x_{n_{k}} \rightarrow x\right\}$. The following lemma is well known.

Lemma 2.3. If a sequence $\left\{x_{n}\right\}$ is bounded and $\lim _{n \rightarrow \infty}\left(x_{n+1}-x_{n}\right)=0$, then

$$
A\left(\left\{x_{n}\right\}\right)=\left[\liminf _{n \rightarrow \infty} x_{n}, \limsup _{n \rightarrow \infty} x_{n}\right] .
$$

Now we analyze the set of accumulation points of $\left\{N_{n}(t, 0) / n\right\}_{n \geq 1}$ for each $t \in \widetilde{C}$. For a fixed $t \in \widetilde{C}$, note that either $N_{n+1}(t, 0)=N_{n}(t, 0)$ or $N_{n+1}(t, 0)=N_{n}(t, 0)+1$. Hence, for any $n \in \mathbb{N}$, we have

$$
-\frac{1}{n+1} \leq \frac{N_{n+1}(t, 0)}{n+1}-\frac{N_{n}(t, 0)}{n} \leq \frac{1}{n+1} .
$$


From Lemma 2.3 it follows that

$$
A\left(\left\{\frac{N_{n}(t, 0)}{n}\right\}\right)=\left[\liminf _{n \rightarrow \infty} \frac{N_{n}(t, 0)}{n}, \limsup _{n \rightarrow \infty} \frac{N_{n}(t, 0)}{n}\right] .
$$

The following lemma is a direct application of the results in [11, Theorem 2] and [12, Corollary 2.4] by Olsen.

Lemma 2.4 (cf. [11, Theorem 2], [12, Corollary 2.4 ]). For $s_{i} \in[0,1]$, $i=1,2,3$, with $s_{1} \leq s_{2}$ let

$$
\begin{aligned}
A_{s_{1}, s_{2}} & =\left\{t \in \widetilde{C}: \liminf _{n \rightarrow \infty} \frac{N_{n}(t, 0)}{n} \leq s_{1} \leq s_{2} \leq \limsup _{n \rightarrow \infty} \frac{N_{n}(t, 0)}{n}\right\}, \\
B_{s_{3}} & =\left\{t \in \widetilde{C}: \limsup _{n \rightarrow \infty} \frac{N_{n}(t, 0)}{n} \leq s_{3}\right\} .
\end{aligned}
$$

Then

$$
\begin{aligned}
\operatorname{dim}_{\mathrm{H}} A_{s_{1}, s_{2}} & =\inf _{p \in\left[s_{1}, s_{2}\right]} \frac{p \log p+(1-p) \log (1-p)}{p \log a_{0}+(1-p) \log a_{1}}, \\
\operatorname{dim}_{\mathrm{P}} A_{s_{1}, s_{2}} & =\xi \\
\operatorname{dim}_{\mathrm{H}} B_{s_{3}} & =\sup _{p \in\left[0, s_{3}\right]} \frac{p \log p+(1-p) \log (1-p)}{p \log a_{0}+(1-p) \log a_{1}} .
\end{aligned}
$$

Proof. Let $E_{1}=\left\{(p, 1-p): p \in\left[s_{1}, s_{2}\right]\right\}, F_{1}=\{(p, 1-p): p \in[0,1]\}$ and $F_{2}=\left\{(p, 1-p): p \in\left[0, s_{3}\right]\right\}$. Then, by (14) and Lemma 2.3, we have

$$
A_{s_{1}, s_{2}}=\left\{t \in \widetilde{C}: E_{1} \subset A\left(\left\{\frac{N_{n}(t, 0)}{n}, \frac{N_{n}(t, 1)}{n}\right\}\right) \subseteq F_{1}\right\}=: D\left(E_{1}, F_{1}\right),
$$

and

$$
B_{s_{3}}=\left\{t \in \widetilde{C}: A\left(\left\{\frac{N_{n}(t, 0)}{n}, \frac{N_{n}(t, 1)}{n}\right\}\right) \subseteq F_{2}\right\}=: D\left(\emptyset, F_{2}\right) .
$$

However, L. Olsen [11, Theorem 2(1), (2)] proved (in a more general setting) that

$$
\begin{aligned}
\operatorname{dim}_{\mathrm{H}} D\left(E_{1}, F_{1}\right) & =\inf _{(p, 1-p) \in E_{1}} \frac{p \log p+(1-p) \log (1-p)}{p \log a_{0}+(1-p) \log a_{1}} \\
\operatorname{dim}_{\mathrm{H}} D\left(\emptyset, F_{2}\right) & =\sup _{(p, 1-p) \in F_{2}} \frac{p \log p+(1-p) \log (1-p)}{p \log a_{0}+(1-p) \log a_{1}}
\end{aligned}
$$

he also proved in [12, Corollary 2.4] (also in a more general setting) that

$$
\operatorname{dim}_{\mathrm{P}} D\left(E_{1}, F_{1}\right)=\sup _{(p, 1-p) \in F_{1}} \frac{p \log p+(1-p) \log (1-p)}{p \log a_{0}+(1-p) \log a_{1}} .
$$

Hence the desired results follow. 
Now we are ready to prove our theorems.

Proof of Theorem 1.1. STEP 1. First we prove that $C \backslash S=S_{0} \cup S_{\infty}$.

Suppose $t \in C \backslash S$. If $t$ is an endpoint of $C$, then it is easy to check that

$$
\liminf _{x \rightarrow t} \frac{F(x)-F(t)}{x-t}=0, \quad \text { and so } \quad t \in S_{0} .
$$

Note that for $x \in \widetilde{C}$ we have

$$
x=\left(1-a_{1}\right) \sum_{k=1}^{\infty} a_{0}^{y(x, k)-k} a_{1}^{k-1} \quad \text { and } \quad F(x)=\sum_{k=1}^{\infty} p_{0}^{y(x, k)-(k-1)} p_{1}^{k-1} .
$$

Let $t \in \widetilde{C} \backslash S$. Take sequences $\left(x_{n}\right)_{n \geq 1}$ and $\left(x_{n}^{*}\right)_{n \geq 1}$ such that

$$
\begin{array}{lll}
y\left(x_{n}, k\right)=y(t, k) \quad \text { for } k<n, & y\left(x_{n}, k\right)=y(t, k)+1 \quad \text { for } k \geq n, \\
y\left(x_{n}^{*}, k\right)=y(t, k) \quad \text { for } k<n, & y\left(x_{n}^{*}, k\right)=y(t, k)+2 \quad \text { for } k \geq n .
\end{array}
$$

Then $\lim _{n \rightarrow \infty} x_{n}=\lim _{n \rightarrow \infty} x_{n}^{*}=t$ and

$$
\begin{aligned}
c & =\lim _{n \rightarrow \infty} \frac{F\left(x_{n}\right)-F(t)}{x_{n}-t}=\lim _{n \rightarrow \infty} \frac{a_{1} p_{0}\left(1-p_{0}\right) \sum_{k=n}^{\infty} p_{0}^{y(t, k)-k} p_{1}^{k}}{p_{1}\left(1-a_{1}\right)\left(1-a_{0}\right) \sum_{k=n}^{\infty} a_{0}^{y(t, k)-k} a_{1}^{k}} \\
& =\lim _{n \rightarrow \infty} \frac{F\left(x_{n}^{*}\right)-F(t)}{x_{n}^{*}-t}=\lim _{n \rightarrow \infty} \frac{a_{1} p_{0}\left(1-p_{0}^{2}\right) \sum_{k=n}^{\infty} p_{0}^{y(t, k)-k} p_{1}^{k}}{p_{1}\left(1-a_{1}\right)\left(1-a_{0}^{2}\right) \sum_{k=n}^{\infty} a_{0}^{y(t, k)-k} a_{1}^{k}} \\
& =\frac{1+p_{0}}{1+a_{0}} c,
\end{aligned}
$$

implying $c \in\{0, \infty\}$, i.e., $t \in S_{0} \cup S_{\infty}$.

SteP 2. For any $p \in\left((s+1)^{-1}, 1\right]$, by Lemma $2.2(\mathrm{I})$ we have

$$
\begin{aligned}
\left\{x \in \widetilde{C}: F^{\prime}(x)=0\right\} & \supseteq\left\{t \in \widetilde{C}: \liminf _{n \rightarrow \infty} \frac{N_{n}(t, 0)}{n}>\frac{1}{s+1}\right\} \\
\supseteq & \left\{t \in \widetilde{C}: \lim _{n \rightarrow \infty} \frac{N_{n}(t, 0)}{n}=p\right\} \\
= & \left\{t \in \widetilde{C}: A\left(\left\{\left(\frac{N_{n}(t, 0)}{n}, \frac{N_{n}(t, 1)}{n}\right)\right\}\right)=(p, 1-p)\right\},
\end{aligned}
$$

implying

$$
\operatorname{dim}_{\mathrm{H}} S_{0} \geq \max _{p \in\left[(s+1)^{-1}, 1\right]} \frac{p \log p+(1-p) \log (1-p)}{p \log a_{0}+(1-p) \log a_{1}} .
$$

STEP 3. We first show that

$$
S_{\infty} \supseteq\left\{t \in \widetilde{C}: 1<\lim _{n \rightarrow \infty} \frac{y(t, n)}{n}<\frac{s+1}{s}\right\}=: T .
$$


It is easy to see (refer to the argument for (8))

$$
\left\{t \in \widetilde{C}: 1<\lim _{n \rightarrow \infty} \frac{y(t, n)}{n}<\frac{s+1}{s}\right\}=\left\{t \in \widetilde{C}: s+1<\lim _{n \rightarrow \infty} \frac{z(t, n)}{n}<\infty\right\} .
$$

Thus, for $t \in T$,

$$
\begin{gathered}
\lim _{n \rightarrow \infty}\left(\frac{z(t, n+1)}{z(t, n)}-\frac{\log a_{1}}{\log p_{1}}+\left(\log \frac{p_{0}}{a_{0}}-\log \frac{p_{1}}{a_{1}}\right) \frac{n}{z(t, n) \log p_{1}}\right) \\
=\lim _{n \rightarrow \infty} \frac{\left(\frac{z(t, n+1)}{n}-s-1\right) \log p_{1}+\left(\frac{z(t, n)}{n}-s-1\right) \log \frac{1}{a_{1}}}{\frac{z(t, n)}{n} \log p_{1}} \\
=\frac{\left(\lim _{n \rightarrow \infty} \frac{z(t, n)}{n}-s-1\right) \log \frac{p_{1}}{a_{1}}}{\log p_{1} \lim _{n \rightarrow \infty} \frac{z(t, n)}{n}}<0 .
\end{gathered}
$$

We claim that (16) implies that

$$
\lim _{x \downarrow t} \frac{F(x)-F(t)}{x-t}=\infty .
$$

In fact, let $k$ be a positive integer such that

$$
\frac{z(t, n+1)}{z(t, n)}-\frac{\log a_{1}}{\log p_{1}}+\left(\log \frac{p_{0}}{a_{0}}-\log \frac{p_{1}}{a_{1}}\right) \frac{n}{z(t, n) \log p_{1}}<q
$$

for some negative real number $q$ whenever $n \geq k$. Let $u$ be the distance between $t$ and $[0,1] \backslash \bigcup_{\omega \in\{0,1\}^{l}} h_{\omega}([0,1])$ with $l=z(t, k)$. Then $u>0$. Let $x$ be a point in the segment $(t, t+u)$. Then $t, x \in h_{\tilde{t} \mid(z(t, n)-1)}([0,1])$, but $x \notin h_{\tilde{t} \mid z(t, n)}([0,1])$ for some $n>k$. Therefore,

$$
\begin{aligned}
\frac{F(x)-F(t)}{x-t} & \geq \frac{p_{1} \prod_{i=1}^{z(t, n+1)-1} p_{t_{i}}}{\prod_{i=1}^{z(t, n)-1} a_{t_{i}}}=\frac{a_{0} p_{1} \prod_{i=1}^{z(t, n+1)} p_{t_{i}}}{p_{0} \prod_{i=1}^{z(t, n)} a_{t_{i}}} \\
& =\frac{a_{0} p_{0}^{n+1} p_{1}^{z(t, n+1)-n}}{p_{0} a_{0}^{n} a_{1}^{z(t, n)-n}} \\
& =a_{0} p_{1}^{z(t, n+1)-z(t, n)}\left(\frac{p_{0}}{a_{0}}\right)^{n}\left(\frac{p_{1}}{a_{1}}\right)^{z(t, n)-n} \\
& =a_{0}\left(p_{1}^{z(t, n+1) / z(t, n)-1}\left(\frac{p_{0}}{a_{0}}\right)^{n / z(t, n)}\left(\frac{p_{1}}{a_{1}}\right)^{1-n / z(t, n)}\right)^{z(t, n)}
\end{aligned}
$$

Let

$$
Q=p_{1}^{z(t, n+1) / z(t, n)-1}\left(\frac{p_{0}}{a_{0}}\right)^{n / z(t, n)}\left(\frac{p_{1}}{a_{1}}\right)^{1-n / z(t, n)}
$$


Taking logs, by (18) we have

$$
\begin{aligned}
\log Q & =\left(\frac{z(t, n+1)}{z(t, n)}-\frac{\log a_{1}}{\log p_{1}}+\left(\log \frac{p_{0}}{a_{0}}-\log \frac{p_{1}}{a_{1}}\right) \frac{n}{z(t, n) \log p_{1}}\right) \log p_{1} \\
& >q \log p_{1}>0 .
\end{aligned}
$$

Since $t$ is a non-endpoint, $z(t, n) \rightarrow \infty$ as $n \rightarrow \infty$ and (17) holds by (19) and (20). On the other hand, for $t \in T$,

$$
\begin{aligned}
\lim _{n \rightarrow \infty}\left(\frac{y(t, n+1)}{y(t, n)}\right. & \left.-\frac{\log a_{0}}{\log p_{0}}+\left(\log \frac{p_{1}}{a_{1}}-\log \frac{p_{0}}{a_{0}}\right) \frac{n}{y(t, n) \log p_{0}}\right) \\
& =\lim _{n \rightarrow \infty} \frac{\left(\frac{y(t, n+1)}{n}-\frac{s+1}{s}\right) \log p_{0}+\left(\frac{y(t, n)}{n}-\frac{s+1}{s}\right) \log \frac{1}{a_{0}}}{\frac{y(t, n)}{n} \log p_{0}} \\
& =\frac{\left(\lim _{n \rightarrow \infty} \frac{y(t, n)}{n}-\frac{s+1}{s}\right) \log \frac{p_{0}}{a_{0}}}{\log p_{0} \lim _{n \rightarrow \infty} \frac{y(t, n)}{n}}<0 .
\end{aligned}
$$

By the same argument as above, this leads to

$$
\lim _{x \uparrow t} \frac{F(x)-F(t)}{x-t}=\infty .
$$

Finally, for each $p \in\left(0,(s+1)^{-1}\right)$ it follows from (15) that

$$
\begin{aligned}
\operatorname{dim}_{\mathrm{H}} S_{\infty} & \geq \operatorname{dim}_{\mathrm{H}}\left\{t \in \widetilde{C}: \lim _{n \rightarrow \infty} \frac{N_{n}(t, 0)}{n}=p\right\} \\
& =\operatorname{dim}_{\mathrm{H}}\left\{t \in \widetilde{C}: A\left(\left\{\left(\frac{N_{n}(t, 0)}{n}, \frac{N_{n}(t, 1)}{n}\right)\right\}\right)=(p, 1-p)\right\} \\
& =\frac{p \log p+(1-p) \log (1-p)}{p \log a_{0}+(1-p) \log a_{1}},
\end{aligned}
$$

implying the desired result.

From the proof of Theorem 1.1 it follows that both $S_{0}$ and $S_{\infty}$ are dense in $C$, and so $\operatorname{dim}_{\mathrm{B}} S_{0}=\operatorname{dim}_{\mathrm{B}} S_{\infty}=\xi$.

Proof of Theorem 1.2. Let $n$ be large enough that $\frac{1}{n}<\min \left\{\frac{1}{s+1}, \frac{s}{s+1}\right\}$. For $k \geq n$ let

$$
\begin{aligned}
& T_{k}=\left\{t \in \widetilde{C}: \liminf _{n \rightarrow \infty} \frac{N_{n}(t, 0)}{n} \leq \frac{1}{s+1}-\frac{1}{k} \leq \frac{1}{s+1}+\frac{1}{k} \leq \limsup _{n \rightarrow \infty} \frac{N_{n}(t, 0)}{n}\right\}, \\
& L_{k}=\left\{t \in \widetilde{C}: \liminf _{n \rightarrow \infty} \frac{N_{n}(t, 0)}{n} \leq \frac{1}{s+1} \leq \frac{1}{s+1}+\frac{1}{k} \leq \limsup _{n \rightarrow \infty} \frac{N_{n}(t, 0)}{n}\right\} .
\end{aligned}
$$


Note that

$$
\begin{gathered}
\left\{t \in \widetilde{C}: \liminf _{n \rightarrow \infty} \frac{N_{n}(t, 0)}{n} \leq \frac{1}{s+1}\right\}=\left\{t \in \widetilde{C}: \limsup _{n \rightarrow \infty} \frac{N_{n}(t, 0)}{n} \leq \frac{1}{s+1}\right\} \\
\cup\left\{t \in \widetilde{C}: \liminf _{n \rightarrow \infty} \frac{N_{n}(t, 0)}{n} \leq \frac{1}{s+1}<\limsup _{n \rightarrow \infty} \frac{N_{n}(t, 0)}{n}\right\} \\
=\left\{t \in \widetilde{C}: \limsup _{n \rightarrow \infty} \frac{N_{n}(t, 0)}{n} \leq \frac{1}{s+1}\right\} \cup \bigcup_{k \geq n} L_{k} .
\end{gathered}
$$

Thus, by (13),

$$
\bigcup_{k \geq n} T_{k} \subseteq S \subseteq\left\{t \in \widetilde{C}: \limsup _{n \rightarrow \infty} \frac{N_{n}(t, 0)}{n} \leq \frac{1}{s+1}\right\} \cup \bigcup_{k \geq n} L_{k} .
$$

From Lemma 2.4 it follows that

$$
\sup _{k \geq n} \operatorname{dim}_{\mathrm{H}} T_{k}=\sup _{k \geq n} \operatorname{dim}_{\mathrm{H}} L_{k}=\frac{s \log s-(s+1) \log (s+1)}{\log a_{0}+s \log a_{1}}, \quad \operatorname{dim}_{\mathrm{P}} T_{k}=\xi,
$$

and

$$
\operatorname{dim}_{\mathrm{H}}\left\{t \in \widetilde{C}: \limsup _{n \rightarrow \infty} \frac{N_{n}(t, 0)}{n} \leq \frac{1}{s+1}\right\}=\frac{s \log s-(s+1) \log (s+1)}{\log a_{0}+s \log a_{1}},
$$

since $1 /(s+1) \leq a_{0}^{\xi}$.

Acknowledgements. Research of W. X. Li was supported by the National Science Foundation of China \#10771071, \#10971069 and Shanghai Leading Academic Discipline Project \#B407.

\section{References}

[1] R. Darst, The Hausdorff dimension of the nondifferentiability set of the Cantor function is $[\ln (2) / \ln (3)]^{2}$, Proc. Amer. Math. Soc., 119 (1993), 105-108.

[2] - Hausdorff dimension of sets of non-differentiability points of Cantor functions, Math. Proc. Cambridge Philos. Soc. 117 (1995), 185-191.

[3] F. M. Dekking and W. X. Li, How smooth is a devil's staircase?, Fractals 11 (2003), 101-107.

[4] K. J. Falconer, Fractal Geometry - Mathematical Foundations and Applications, Wiley, Chichester, 1990.

[5] - One-sided multifractal analysis and points of non-differentiability of devil's staircases, Math. Proc. Cambridge Philos. Soc. 136 (2004), 167-174.

[6] J. E. Hutchinson, Fractals and self-similarity, Indiana Univ. Math. J. 30 (1981), $713-747$.

[7] W. X. Li, D. M. Xiao and F. M. Dekking, Non-differentiability of devil's staircases and dimensions of subsets of Moran fractals, Math. Proc. Cambridge Philos. Soc. 133 (2002), 345-355.

[8] W. X. Li, Points of infinite derivative of Cantor functions, Real Anal. Exchange 32 (2006/07), 87-96. 
[9] W. X. Li, Non-differentiability points of Cantor functions, Math. Nachr. 280 (2007), 140-151.

[10] J. Morris, The Hausdorff dimension of the nondifferentiability set of a nonsymmetric Cantor function, Rocky Mountain J. Math. 32 (2002), 357-370.

[11] L. Olsen, A generalization of a result by W. Li and F. Dekking on the Hausdorff dimension of subsets of self-similar sets with prescribed group frequency of their codings, Aequationes Math. 72 (2006), 10-26.

[12] - Multifractal analysis of divergence points of deformed measure theoretical Birkhoff averages. IV: Divergence points and packing dimension, Bull. Sci. Math. 132 (2008), 650-678.

Department of Mathematics

East China Normal University

Shanghai 200241, P.R. China

E-mail: wxli@math.ecnu.edu.cn

Received February 23, 2008

Revised version July 4, 2009 